\title{
Glucose-lowering agents and reduced risk of incident non-alcoholic fatty liver disease: new insights
}

\author{
Alessandro Mantovani ${ }^{1}$, Rosa Lombardi ${ }^{2,3}$, Andrea Dalbeni ${ }^{4}$ \\ ${ }^{1}$ Section of Endocrinology, Diabetes and Metabolism, Department of Medicine, University and Azienda Ospedaliera Universitaria Integrata of \\ Verona, Verona, Italy; ${ }^{2}$ Unit of Internal Medicine and Metabolic Disease, Fondazione IRCCS Ca' Granda Ospedale Maggiore Policlinico, Milan, Italy; \\ ${ }^{3}$ Department of Pathophysiology and Transplantation, Università degli Studi di Milano, Milan, Italy; ${ }^{4}$ Section of General Medicine C and Liver Unit, \\ University and Azienda Ospedaliera Universitaria Integrata of Verona, Verona, Italy \\ Correspondence to: Dr. Alessandro Mantovani, MD, PhD. Department of Medicine, University and Azienda Ospedaliera Universitaria Integrata of \\ Verona, 37126 Verona, Italy. Email: alessandro.mantovani@univr.it. \\ Comment on: van Dalem J, Driessen JHM, Burden AM, et al. Thiazolidinediones and Glucagon-Like Peptide-1 Receptor Agonists and the Risk of \\ Nonalcoholic Fatty Liver Disease: A Cohort Study. Hepatology 2021;74:2467-77.
}

Submitted Dec 09, 2021. Accepted for publication Dec 28, 2021.

doi: $10.21037 / \mathrm{hbsn}-2021-28$

View this article at: https://dx.doi.org/10.21037/hbsn-2021-28

Non-alcoholic fatty liver disease (NAFLD) is classically defined as the hepatic manifestation of metabolic syndrome (1). Histologically, NAFLD covers a range of conditions, spanning from simple steatosis to non-alcoholic steatohepatitis (NASH) and cirrhosis (2). At present, NAFLD is the most frequent chronic liver disease seen in clinical practice in high-income countries, as it affects nearly $30 \%$ of adults in the general population, up to $70 \%$ of patients with type 2 diabetes (T2DM) and almost all patients with obesity (2).

Over the last decade, it has become evident that NAFLD is a "multisystemic" disease (2), which is not only associated with hepatic complications [i.e., hepatocellular carcinoma (HCC)], but also with a higher risk of extra-hepatic complications, including cardiovascular disease (CVD) and T2DM (2).

In spite of the clinical burden of NAFLD, there are no pharmacological treatments for NAFLD/NASH approved by main national medicines agencies to date (3). However, based on the data provided by randomized controlled trials (RCTs) (Table 1), the EASL-EASD-EASO and AASLD practice guidelines for the NAFLD management now recommend the use of pioglitazone (which is a member of thiazolidinediones) in non-cirrhotic adults with biopsyconfirmed NASH $(4,5)$. That said, several other drugs have been tested in phase-2 RCTs as potential therapy for NAFLD/NASH (3). Among these, glucagon-like peptide-1 receptor agonists (GLP-1RAs) have received attention, as these agents are able to promote weight loss and to improve glycemic control and insulin resistance (3). In a 2021 metaanalysis, for instance, Mantovani et al. (6) reported that, compared to control therapy, treatment with GLP-1RAs was associated with reductions in the absolute percentage of liver fat content (as assessed by magnetic resonance-based techniques), as well as with histological resolution of NASH without worsening of liver fibrosis.

However, to date, information on the association between the use of specific agents (including thiazolidinediones or GLP-1RAs) and the risk of developing NAFLD and its advanced forms is scarce. Notably, understanding this issue could help to create novel therapeutic strategies that may reduce the clinical burden of NAFLD, which is strongly associated with an excess of premature mortality (2).

Recently, on Hepatology, van Dalem et al. (7) published the results of a large population-based cohort study collecting primary care data from the Clinical Practice Research Datalink database (2007-2018) with the aim to assess the potential role of different glucose-lowering agents on the risk of incident NAFLD. In this study involving 207,367 UK T2DM adults [median age: 61 years; $45 \%$ women; median body mass index (BMI): $31 \mathrm{~kg} / \mathrm{m}^{2}$ ] with an initial prescription of glucose-lowering agents, the investigators found that 2,526 patients had a new diagnosis of NAFLD (as assessed by Read codes) during 


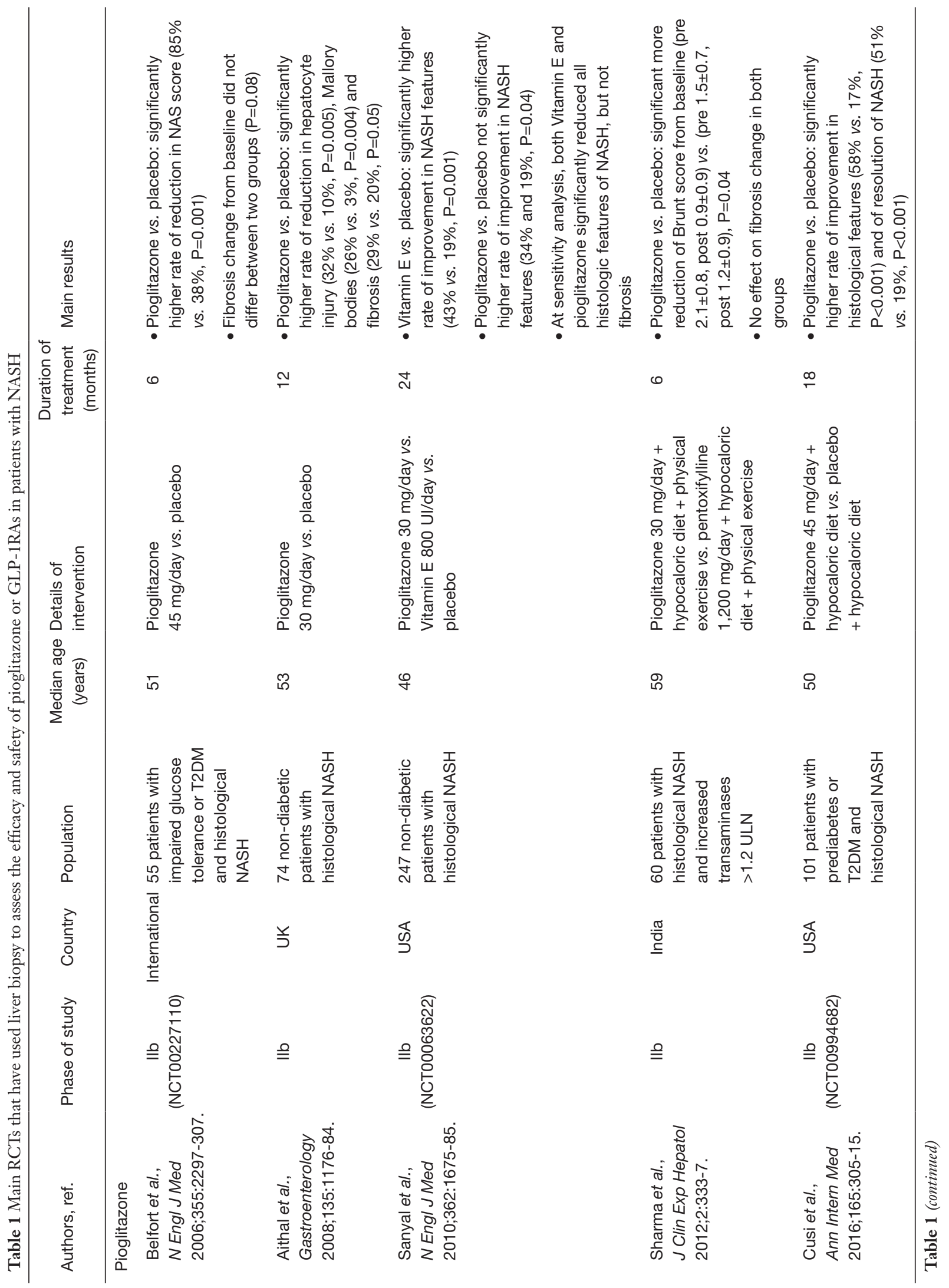




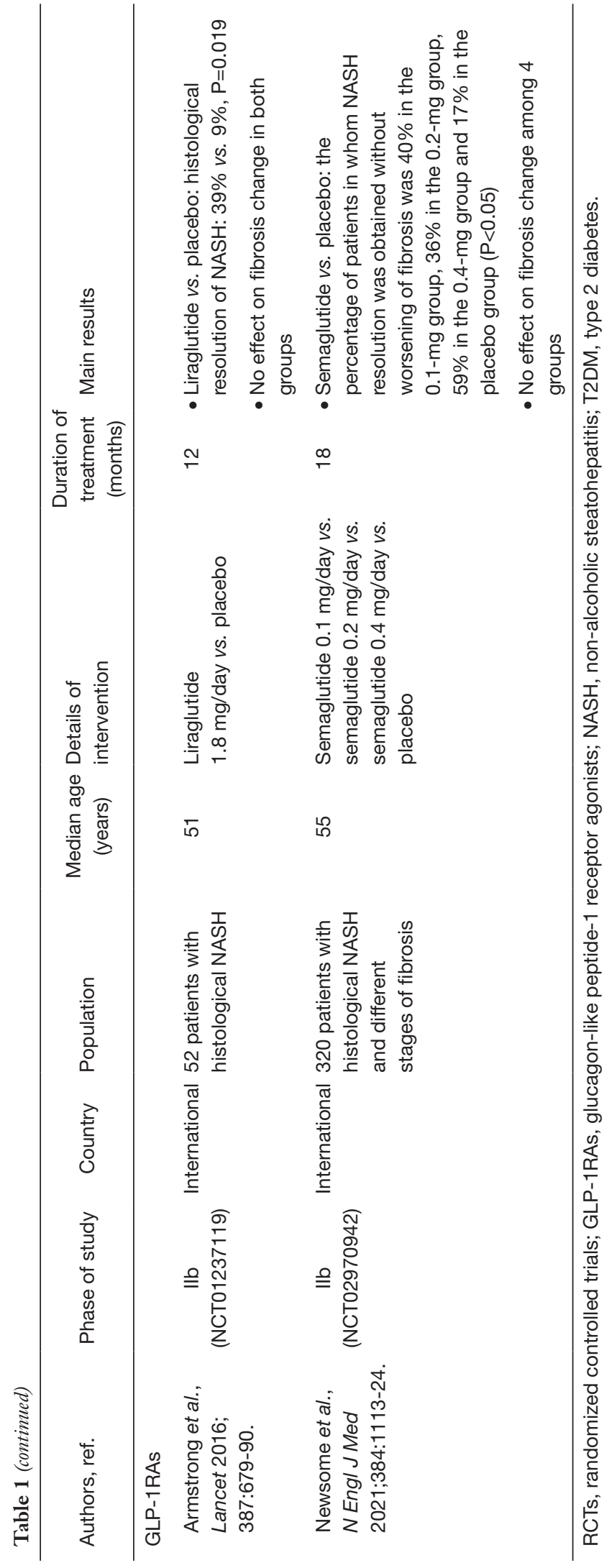

a mean follow-up of nearly 5 years (7). Interestingly, the risk of developing NAFLD was lower in patients receiving thiazolidinediones (mainly pioglitazone) compared with those receiving sulphonylureas, even after adjustment for age, sex, BMI, HbAlc and use of glucocorticoids (adjustedHR: 0.32 ; $95 \%$ confidence interval: $0.20-0.51)$ (7). Conversely, no difference in the risk of incident NAFLD was observed between patients receiving GLP-1RAs and those receiving insulin therapy (adjusted-HR: 1.22; 95\% confidence interval: 0.91-1.63) (7). The investigators concluded that their study further endorses the use of thiazolidinediones (pioglitazone) in specific patients at risk of developing NAFLD (7), thereby suggesting that these agents may reduce the clinical burden of NAFLD.

We believe that van Dalem et al. (7) should be congrats for their interesting findings. Indeed, their study confirmed, but also expanded, the findings of previous RCTs and meta-analyses (3), suggesting that pioglitazone is able to promote the resolution of NASH and to improve liver fibrosis. However, pioglitazone is not yet approved by most national medicines agencies outside the use for the treatment of T2DM, and, therefore, the off-label use of this agent for NAFLD/NASH treatment requires the patient's consent. Interestingly, pioglitazone can also reduce the risk of developing cardiovascular events (8), which are the primary cause of death amongst NAFLD patients (2). However, concerns about its side-effects (i.e., weight gain, fluid retention, risk of bone fractures) might preclude the use of pioglitazone in clinical practice. Hence, other agents have been studied as potential therapy for NAFLD.

The history of GLP-1RAs for the treatment of NAFLD/ $\mathrm{NASH}$ is instead at the beginning and, unfortunately, the study by van Dalem et al. (7) does not allow to draw firm conclusions on this topic for the following reasons. First of all, it is an observational study and, hence, it cannot establish the causality of the observed findings. Second, the results of the study by van Dalem et al. (7) may be potentially influenced by specific biases (including the "prescription" bias) that can be only partially overcome by advanced statistical analyses. In this regard, it is important to note that, in real life, insulin therapy is usually prescribed to patients with advanced stage of T2DM, whereas GLP1RAs is often prescribed to T2DM patients with obesity. Third, patients included in the study by van Dalem et al. (7) had T2DM and, hence, the results cannot be generalized to other patient populations. Lastly, the diagnosis of NAFLD was made by Read codes that can potentially lead to misclassification of NAFLD and, even, under-recording it. 
In this context, it is important to remember that at present, although specific indirect markers are being studied, the gold-standard method for the diagnosis of NAFLD and its advanced forms is still liver biopsy $(4,5)$.

Presently, as reported in Table 1, there are only two published phase-IIb RCTs $(9,10)$ that have used liver biopsy to determine the efficacy of GLP-1RAs in patients with NAFLD/NASH. Notably, the resolution of NASH and/or improvement in fibrosis stage are the two main histological endpoints requested by drug regulatory agencies for approval of NAFLD/NASH pharmacotherapies. In a 48-week, double-blinded RCT enrolling 52 patients with biopsy-proven NASH randomly to receive liraglutide $(n=26)$ or placebo $(n=26)$, Armstrong et al. (9) showed that liraglutide led to the resolution of NASH (9). Conversely, liraglutide did not lead to the improvement of liver fibrosis. In a recent 72 -week, double-blind RCT involving 320 patients with biopsy-confirmed NASH and liver fibrosis randomly to receive semaglutide at a dose of $0.1 \mathrm{mg}(\mathrm{n}=80), 0.2 \mathrm{mg}(\mathrm{n}=78)$, or $0.4 \mathrm{mg}(\mathrm{n}=82)$ or to receive placebo $(\mathrm{n}=80)$, Newsome et al. (10) showed that the percentage of patients in whom NASH resolution was observed with no worsening of fibrosis was $40 \%$ in the $0.1-\mathrm{mg}$ group, $36 \%$ in the $0.2-\mathrm{mg}$ group, $59 \%$ in the $0.4-\mathrm{mg}$ group, and $17 \%$ in the placebo group $(\mathrm{P}<0.05)$. Again, this RCT did not report between-group differences in the percentage of patients who experienced an improvement in fibrosis stage without worsening fibrosis as well (10). However, some relevant aspects need to be discussed regarding these findings. First, the most individuals included in these trials had NAFLD and T2DM. Hence, additional trials in nondiabetic individuals with NAFLD are required. Second, in the trial by Newsome et al. (10) the patients with biopsy-proven NAFLD were randomly assigned to receive once-daily semaglutide at a dose of $0.1,0.2,0.4 \mathrm{mg}$ or placebo. This is not the current approved dosage of semaglutide for the treatment of T2DM. Third, the insufficient follow-up duration might have contributed to not demonstrating a beneficial effect of GLP-RAs on liver fibrosis. For these reasons, additional trials are timely needed to test the exact efficacy of GLP1RAs in patients with NAFLD/NASH and to establish if these glucose-lowering agents may reduce the risk of developing NAFLD/NASH over time.

Maybe, given the multiple pathways involved in the NAFLD pathogenesis and the single response from singleagent therapies (that stands from $30 \%$ to $50 \%$ ) observed in the RCTs published so far (3), the combination of different agents with various mechanisms of action may be the best way to treat NAFLD and its advanced forms (11). In this regard, for instance, the GLP-1 RA, semaglutide, is being investigated in combination with the nonsteroidal Farnesoid $\mathrm{X}$ receptor (FXR) agonist, cilofexor, and with the acetylCoA carboxylase inhibitor, firsocostat, in a phase 2 proofof-concept trial (NCT03987074). Semaglutide is also being investigated in combination with empagliflozin (gliflozin) in a placebo-controlled, double-blind, randomized, 3-arm parallel group trial (NCT04639414). Moreover, considering the heterogeneity of NAFLD patients, it might be even more suitable to identify specific individuals for a definite therapeutic strategy (11). However, the research on this topic is still at the beginning and further studies are needed to improve our understanding for intercepting NAFLD patients who would have a higher probability of treatment response with a specific agent as monotherapy or, better, with a combination therapy (3).

\section{Acknowledgments}

Funding: None.

\section{Footnote}

Provenance and Peer Review: This article was commissioned by the editorial office, Hepatobiliary Surgery and Nutrition. The article did not undergo external peer review.

Conflicts of Interest: All authors have completed the ICMJE uniform disclosure form (available at https://hbsn. amegroups.com/article/view/10.21037/hbsn-2021-28/coif). The authors have no conflicts of interest to declare.

Ethical Statement: The authors are accountable for all aspects of the work in ensuring that questions related to the accuracy or integrity of any part of the work are appropriately investigated and resolved.

Open Access Statement: This is an Open Access article distributed in accordance with the Creative Commons Attribution-NonCommercial-NoDerivs 4.0 International License (CC BY-NC-ND 4.0), which permits the noncommercial replication and distribution of the article with the strict proviso that no changes or edits are made and the original work is properly cited (including links to both the formal publication through the relevant DOI and the license). See: https://creativecommons.org/licenses/by-nc-nd/4.0/. 


\section{References}

1. Sheka AC, Adeyi O, Thompson J, et al. Nonalcoholic Steatohepatitis: A Review. JAMA 2020;323:1175-83.

2. Mantovani A, Scorletti E, Mosca A, et al. Complications, morbidity and mortality of nonalcoholic fatty liver disease. Metabolism 2020;111S:154170.

3. Mantovani A, Dalbeni A. Treatments for NAFLD: State of Art. Int J Mol Sci 2021;22:2350.

4. European Association for the Study of the Liver (EASL); European Association for the Study of Diabetes (EASD); European Association for the Study of Obesity (EASO). EASL-EASD-EASO Clinical Practice Guidelines for the management of non-alcoholic fatty liver disease. J Hepatol 2016;64:1388-402.

5. Chalasani N, Younossi Z, Lavine JE, et al. The diagnosis and management of nonalcoholic fatty liver disease: Practice guidance from the American Association for the Study of Liver Diseases. Hepatology 2018;67:328-57.

6. Mantovani A, Petracca G, Beatrice G, et al. GlucagonLike Peptide-1 Receptor Agonists for Treatment of Nonalcoholic Fatty Liver Disease and Nonalcoholic Steatohepatitis: An Updated Meta-Analysis of Randomized
Controlled Trials. Metabolites 2021;11:73.

7. van Dalem J, Driessen JHM, Burden AM, et al. Thiazolidinediones and Glucagon-Like Peptide-1 Receptor Agonists and the Risk of Nonalcoholic Fatty Liver Disease: A Cohort Study. Hepatology 2021;74:2467-77.

8. DeFronzo RA, Inzucchi S, Abdul-Ghani M, et al. Pioglitazone: The forgotten, cost-effective cardioprotective drug for type 2 diabetes. Diab Vasc Dis Res 2019;16:133-43.

9. Armstrong MJ, Gaunt P, Aithal GP, et al. Liraglutide safety and efficacy in patients with non-alcoholic steatohepatitis (LEAN): a multicentre, double-blind, andomized, placebo-controlled phase 2 study. Lancet 2016;387:679-90.

10. Newsome PN, Buchholtz K, Cusi K, et al. A PlaceboControlled Trial of Subcutaneous Semaglutide in Nonalcoholic Steatohepatitis. N Engl J Med 2021;384:1113-24.

11. Dufour JF, Caussy C, Loomba R. Combination therapy for non-alcoholic steatohepatitis: rationale, opportunities and challenges. Gut 2020;69:1877-84.

Cite this article as: Mantovani A, Lombardi R, Dalbeni A. Glucose-lowering agents and reduced risk of incident nonalcoholic fatty liver disease: new insights. HepatoBiliary Surg Nutr 2022;11(1):156-160. doi: 10.21037/hbsn-2021-28 